\title{
A network pharmacology approach to establish the pharmacological mechanism of JiaWeiXianJiTang on inflammatory bowel disease
}

\author{
LING-JIA TAO ${ }^{1}$, WEN-TING CHEN ${ }^{1}$, LIN JING $^{1}$, QING JI $^{2}$ and JIAN-LIN REN ${ }^{1}$ \\ ${ }^{1}$ Department of Medical Oncology, Shanghai Municipal Hospital of Traditional Chinese Medicine, \\ Shanghai University of Traditional Chinese Medicine, Shanghai 200071; ${ }^{2}$ Department of Medical Oncology, \\ Shuguang Hospital, Shanghai University of Traditional Chinese Medicine, Shanghai 201203, P.R. China
}

Received November 10, 2016; Accepted January 9, 2017

DOI: $10.3892 /$ br.2017.853

\begin{abstract}
The aim of the present study was to characterize the molecular mechanism of the effective components of JiaWeiXianJiTang (JWXJT), a traditional Chinese medicine, on inflammatory bowel disease (IBD) using network pharmacology technology. Data regarding natural molecules of JWXJT, targets of IBD, and interactions between natural molecules and IBD targets were screened. Network pharmacology of the interactions between natural molecules and IBD targets were drawn using Cytoscape v3.2.1. As a result of screening, 205 interactions were identified between 673 natural molecules and 76 targets of IBD. By analyzing the effective components, the complex effect mechanism of JWXJT on IBD was identified to be via enhancement of the immune system, inhibiting novel blood vessel growth through 15-hydroxyprostaglandin dehydrogenase, 5' AMP-activated protein kinase, interleukin-2 and macrophage colony-stimulating factor-1, and resulting in inflammation inhibition. In addition, JWXJT ameliorated IBD by antagonizing various molecular targets of IBD through
\end{abstract}

Correspondence to: Professor Jian-Lin Ren, Department of Medical Oncology, Shanghai Municipal Hospital of Traditional Chinese Medicine, Shanghai University of Traditional Chinese Medicine, 274 Zhijiang Middle Road, Shanghai 200071, P.R. China E-mail: renjianlin666@126.com

Mr. Qing Ji, Department of Medical Oncology, Shuguang Hospital, Shanghai University of Traditional Chinese Medicine, 528 Zhangheng Road, Shanghai 201203, P.R. China

E-mail: ttt99118@hotmail.com

Abbreviations: JWXJT, JiaWeiXianJiTang; IBD, inflammatory bowel disease; TCM, traditional Chinese medicine; UC, ulcerative colitis; CD, Crohn's disease; CAC, colitis-associated cancer

Key words: JiaWeiXianJiTang, inflammatory bowel disease, network pharmacology, pharmacological mechanism its effective components, inhibiting inflammatory reactions, improving patient quality of life, as well as reducing the incidence of cancer.

\section{Introduction}

Tumor-promoting inflammation, recognized as the eighth hallmark of cancer (1), is highly involved in tumor growth, invasion and metastasis. Inflammatory bowel diseases (IBDs), including ulcerative colitis (UC) and Crohn's disease (CD), are associated with chronic relapsing inflammation of the intestinal tract of unknown etiology (2). IBD is somewhat common in western countries, with a prevalence rate of 100-200/100,000. Over the past 10 years, the incidence of IBD has increased by approximately 10-20 times in China (3). Given the serious harm and higher malignant transformation probability associated with IBD, increasing numbers of individuals are becoming concerned that this disease is a type of precancerous lesion (4). Currently, it is proposed that IBD is caused by the multifactorial interactions between environment, gene, infection and immune disorders (5). Activation of the intestinal immune system and non-specific immune system, which leads to immunoreaction and the inflammatory response, is important in the pathogenesis of IBD.

XianJiTang, which has marked curative effects on chronic diarrhoea, was created by Zhu et al (6). Long-term clinical practice and research have demonstrated its efficacy $(7,8)$. JiaWeiXianJiTang (JWXJT), which is comprised of XianJiTang and JianPi Chinese medicine, is administered to patients for diarrhoea, colorectal cancer or advanced colorectal cancer surgery. As they are inherently complex and are comprised of numerous components, a characteristic of traditional Chinese medicine (TCM) recipes is that they affect multiple targets and interact with other herbal medicines. Studies investigating TCM at the cell and molecular levels are difficult to conduct due to the unknown effective components and mechanisms of action, as well as the unstable herbal quality. The present study analyzed the effective components of JWXJT and the therapeutic targets of IBD, and constructed a relational network between them. Based on the results of network analysis, the therapeutic targets of IBD and the mechanisms of JWXJT 
were characterized at the cell and molecular levels, and the regulatory interactions were analyzed in their entirety.

\section{Materials and methods}

Data preparation. The JWXJT was composed as follows: Agrimonia pilosa var. japonica (30 g), Platycodon grandiflorum (8 g), Coix lacryma-jobi (30 g), Dolichos lablab (15 g), Plantago asiatica (15 g), Codonopsis pilosula (9 g), Poria cocos (9 g), Paeonia albiflora (9 g), Areca catechu (1.2 g), Saussurea lappa (5 g), Hibiscus syriacus (9 g), Prunus mume $(4.5 \mathrm{~g})$ and Glycyrrhiza uralensis $(4.5 \mathrm{~g})$. It is proposed that patients with colorectal cancer that have not undergone surgery and postoperative patients with colorectal cancer who are susceptible to IBD, present with a deficiency of the spleen and kidney, as well as symptoms, including an elevated heat, dampness, phlegm and blood stasis. Deficiencies of the spleen and kidney exist throughout the course of IBD, being significant in its initiation and progression (9).

TCM-potential target database (PTD). The TCM-PTD (http://pharminfo.zju.edu.cn/ptd), which is dedicated to providing accurate potential targets of TCM that are predicted using state-of-the-art machine learning approaches, comprising three databases 'compounds', 'target', and 'total-relationship'. The above three databases describe the sources of the compounds, targets, as well as the interaction between compounds and targets of the herbs.

Bibliographic database. The present study used the following databases: The Wanfang Database (http://www.wanfangdata.com/), China National Knowledge Infrastructure (CNKI; http://www.global.cnki.net/), and the VIP Database (http://www.cqvip.com/). The following search terms were used: 'Inflammatory bowel disease' or 'enteritis' or 'target'.

Data analyses. The compounds of each herb in JWXJT and the targets of IBD were screened in the TCM-PTD database. Following virtual screening in the TCM-PTD database, discarding the repetitive data and the data where the docking scores were less than five, the final data were applied for further network construction. Network construction was performed as follows: i) A compound-compound target network was established by linking chemical compounds and corresponding targets; ii) a herb-compound target-IBD target network was constructed by connecting the 13 JWXJT herbs, the corresponding compound targets, and the IBD targets that interacted with the compound targets. The networks were generated using the network visualization software Cytoscape version 3.2.1 (http://cytoscape.org/), which is used to visualize biological pathways and networks of molecular interactions, and to interact with these networks via profiles of gene expression, annotations, as well as other state data. The software then offers a basic set of features for data integration, analysis and visualization for complicated network analysis.

\section{Results}

Drug targets associated with IBD. Four hundred and twenty-one, 410 and 58 articles were retrieved from the
Table I. Articles associated with the targets of inflammatory bowel disease.

\begin{tabular}{lccc}
\hline & \multicolumn{3}{c}{ Articles (n) } \\
\cline { 2 - 4 } Database & $\begin{array}{c}\text { 'Target' and 'Target' and } \\
\text { 'IBD' }\end{array}$ & 'enteritis' & Total \\
\hline Wanfang Database & 126 & 295 & 421 \\
$\begin{array}{l}\text { China National } \\
\text { Knowledge Infrastructure }\end{array}$ & 155 & 255 & 410 \\
VIP Database & 28 & 30 & 58 \\
\hline
\end{tabular}

IBD, inflammatory bowel disease.

Wanfang Database, CNKI and VIP Database, respectively (Table I). The targets of IBD associated with JWXJT were summarized as follows: 15-Hydroxyprostaglandin dehydrogenase (15-PGDH), 5' AMP-activated protein kinase (AMPK), bradykinin receptor, cellular tumor antigen p53, cysteinyl leukotriene receptor (CYSLTR), dual specificity mitogen-activated protein kinase (MAPK), eosinophil cationic protein, glutathione-requiring prostaglandin D synthase, macrophage colony-stimulating factor (M-CSF), nitric oxide (NO), prostaglandin E2 receptor EP4 subtype (PTGE), prostaglandin reductase 2, transforming growth factor (TGF) and tumor necrosis factor (TNF; Fig. 1).

Compound-compound target network analysis. The compound-compound target network is shown in Fig. 2, and includes 205 interactions between 673 compounds in JWXJT and 76 compound targets. In the network, certain targets demonstrated more interactions with compounds than others. This indicated that a large number of targets may be regulated by multiple compounds rather than just one. For example, M-CSF and PGDH were regulated by multiple JWXJT ingredients, including Saussurea lappa and Glycyrrhiza uralensis. In addition, AMPK, interleukin (IL), CYSLTR, MAPK, NO, PTGE, prostaglandin reductase 2 and TGF are regulated by more than one compound.

Herb compound target-IBD target network analysis. The herb compound target-IBD target network was constructed to identify the interactions between 13 herbs in JWXJT, and the corresponding compound targets and IBD targets. The network was composed of 205 interactions (13 herbs, 673 compound targets and 76 IBD targets; Fig. 3). Glycyrrhiza uralensis demonstrated the highest degree of distribution followed by Saussurea lappa, Codonopsis pilosula and Poria cocos, the interactions of which with other herbs were more than one, thus, demonstrating their significance in the network.

Glycyrrhiza uralensis and Codonopsis pilosula regulated multiple targets, including $\mathrm{M}-\mathrm{CSF}-1$ receptor and PGDH (Fig. 4). Furthermore, Agrimonia pilosa var. japonica, Platycodon grandiflorum, Paeonia albiflora, Areca catechu, Saussurea lappa, Hibiscus syriacus, Prunus mume, Plantago asiatica, Coix lacryma-jobi, Dolichos lablab and Poria cocos also regulated more than one target. 


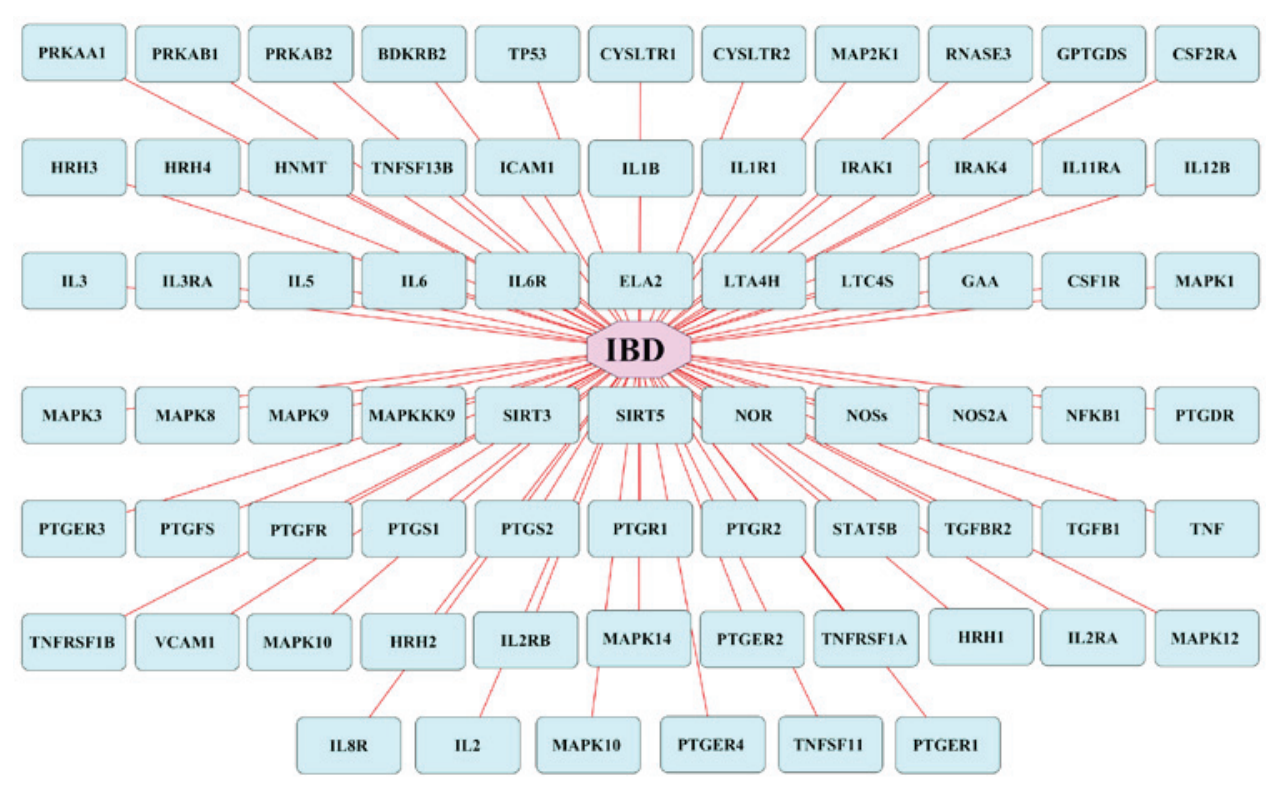

Figure 1. Multiple targets of IBD. The blue rectangles represent the targets associated with IBD. IBD, inflammatory bowel disease; 15-PGDH, 15-hydroxyprostaglandin dehydrogenase; AMPK, 5' AMP-activated protein kinase; BDKRB2, bradykinin receptor, cellular tumor antigen p53; CYSLTR, cysteinyl leukotriene receptor; MAPK, dual specificity mitogen-activated protein kinase; RNASE3, eosinophil cationic protein; GPTGDS, glutathione-requiring prostaglandin D synthase; M-CSF, macrophage colony-stimulating factor; NO, nitric oxide; PTGE, prostaglandin E2 receptor EP4 subtype; PTGR2, prostaglandin reductase 2; TGF, transforming growth factor; TNF, tumor necrosis factor.

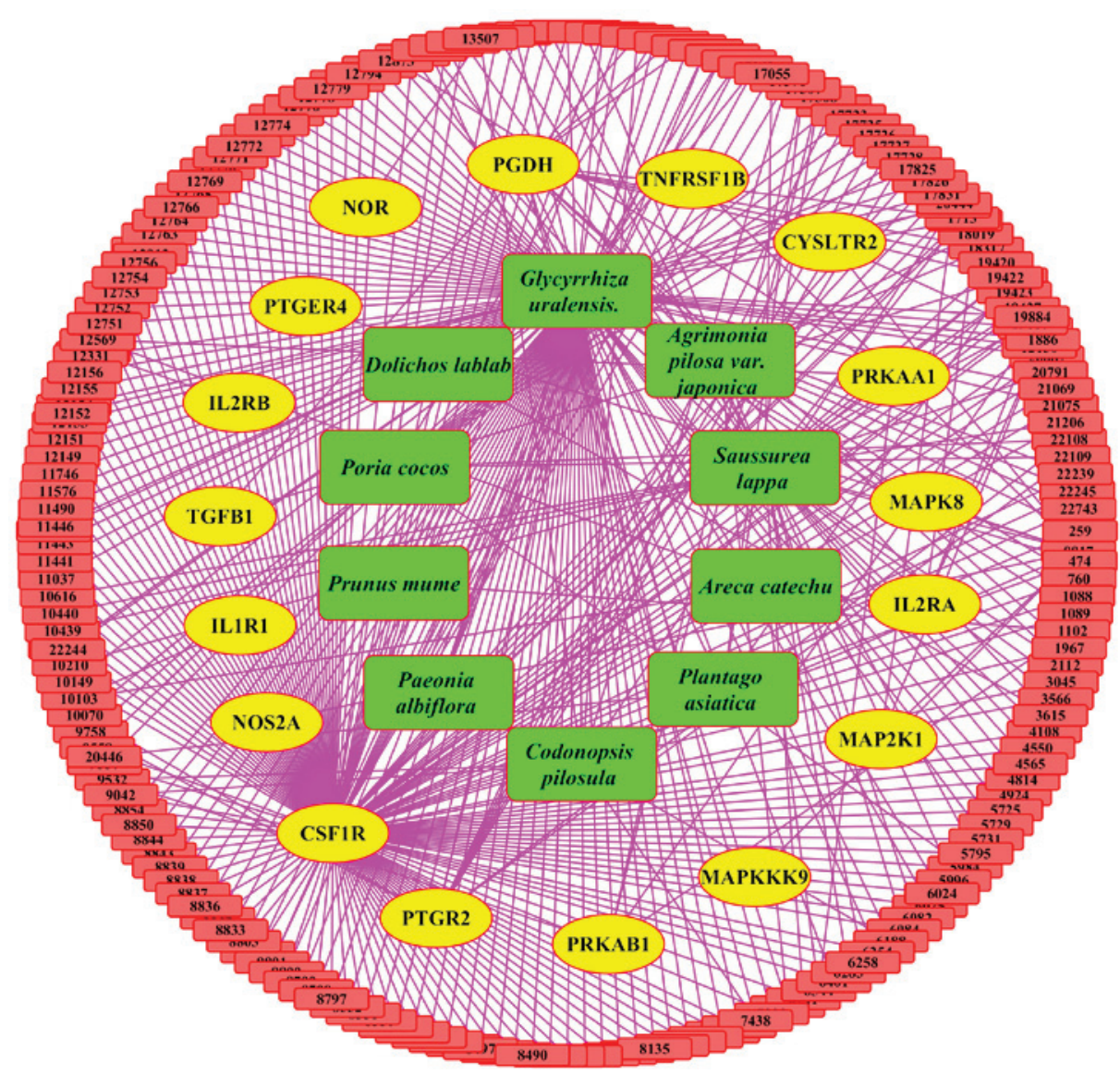

Figure 2. Compound-compound target network construction. Green rectangles represent the ingredients of JWXJT, the yellow circles represent the predicted targets, and the red rectangles represent the multiple compounds of JWXJT. JWXJT, JiaWeiXianJiTang. 15-PGDH, 15-hydroxyprostaglandin dehydrogenase; PRKAA1, 5'-AMP-activated protein kinase catalytic subunit $\alpha$-1; PRKAB1, 5'-AMP-activated protein kinase subunit $\beta$-1; CYSLTR2, cysteinyl leukotriene receptor 2; MAP2K1, dual specificity mitogen-activated protein kinase kinase 1; IL1R1, interleukin-1 receptor type I; IL2RA, interleukin-2 receptor $\alpha$ chain; IL2RB, interleukin-2 receptor subunit $\beta$; CSF1R, macrophage colony-stimulating factor 1 receptor; MAPK8, mitogen-activated protein kinase 8; MAPKKK9, mitogen-activated protein kinase kinase kinase 9; NOR, nitric oxide reductase; NOS2A, nitric oxide synthase, inducible; PTGER4, prostaglandin E2 receptor EP4 subtype; PTGR2, prostaglandin reductase 2; TGFB1, transforming growth factor $\beta$-1; TNFRSF1B, tumor necrosis factor receptor superfamily member $1 \mathrm{~B}$. 


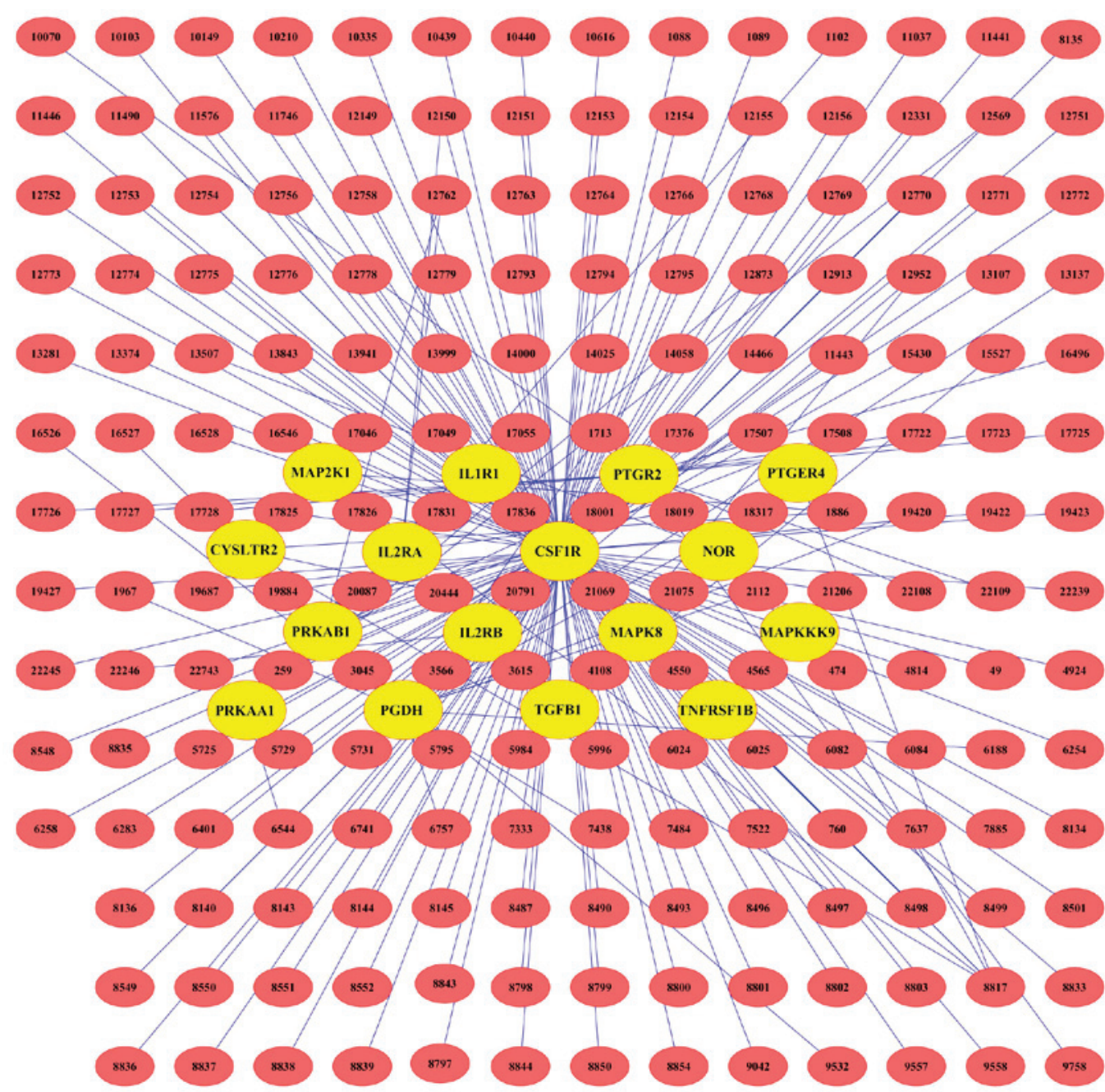

Figure 3. Herb compound target-IBD target network construction. Red rectangles represent the multiple compounds of JWXJT and the yellow circles represent the IBD targets. IBD, inflammatory bowel disease; JWXJT, JiaWeiXianJiTang. 15-PGDH, 15-hydroxyprostaglandin dehydrogenase; PRKAA1, 5'-AMP-activated protein kinase catalytic subunit $\alpha$-1; PRKAB1, 5'-AMP-activated protein kinase subunit $\beta$-1; CYSLTR2, cysteinyl leukotriene receptor 2; MAP2K1, dual specificity mitogen-activated protein kinase kinase 1; IL1R1, interleukin-1 receptor type I; IL2RA, interleukin-2 receptor $\alpha$ chain; IL2RB, interleukin-2 receptor subunit $\beta$; CSF1R, macrophage colony-stimulating factor 1 receptor; MAPK8, mitogen-activated protein kinase 8; MAPKKK9, mitogen-activated protein kinase kinase kinase 9; NOR, nitric oxide reductase; PTGER4, prostaglandin E2 receptor EP4 subtype; PTGR2, prostaglandin reductase 2; TGFB1, transforming growth factor $\beta-1$; TNFRSF1B, tumor necrosis factor receptor superfamily member $1 \mathrm{~B}$.

\section{Discussion}

In the present study, the potential molecular mechanism of effective components of the TCM, JWXJT on IBD were investigated using network pharmacology technology. JWXJT is composed of Agrimonia pilosa var. japonica, Platycodon grandiflorum, Coix lacryma-jobi, Dolichos lablab, Plantago asiatica, Codonopsis pilosula, Poria cocos, Paeonia albiflora, Areca catechu, Saussurea lappa, Hibiscus syriacus, Prunus mume and Glycyrrhiza uralensis. Compounds from Saussurea lappa and Glycyrrhiza uralensis have the greatest number of targets. It was hypothesized that they were the most important components of JWXJT, presenting as the monarch herbs according to 'Sovereign and subject Musa acts'. This refers to monarch, minister, assistant and guide, which describe the status of different herbs in the TCM prescription, as well as the variations in compatibility of different herbs, from the perspective of multi-drug usage. Monarch herbs are those in the prescription that exert the predominant therapeutic effect on the disease/TCM syndromes. These are considered to be the indispensable prescription herbs, which perform the main actions, and exert the initial and most important effect (10). Compounds from Codonopsis pilosula and Poria cocos also have numerous targets, potentially the less important components in JWXJT, representing the minister herbs (that support the monarch herbs in the treatment of the primary disease/TCM syndromes). Compounds from Agrimonia pilosa var. japonica, Paeonia albiflora, Areca catechu, Prunus mume and Dolichos lablab had the fewest targets. It could be speculated that they are the accessory components in JWXJT, serving as the assistant and guide herbs. Compounds from Platycodon grandiflorum, Coix lacryma-jobi and Hibiscus syriacus did not have any targets, although these three herbs are often administered in clinical TCM treatment for IBD or other diseases. The reason for this deficiency of targets may be due to the insufficient data capacity of the TCM-PTD or that the data was not up-to-date. 


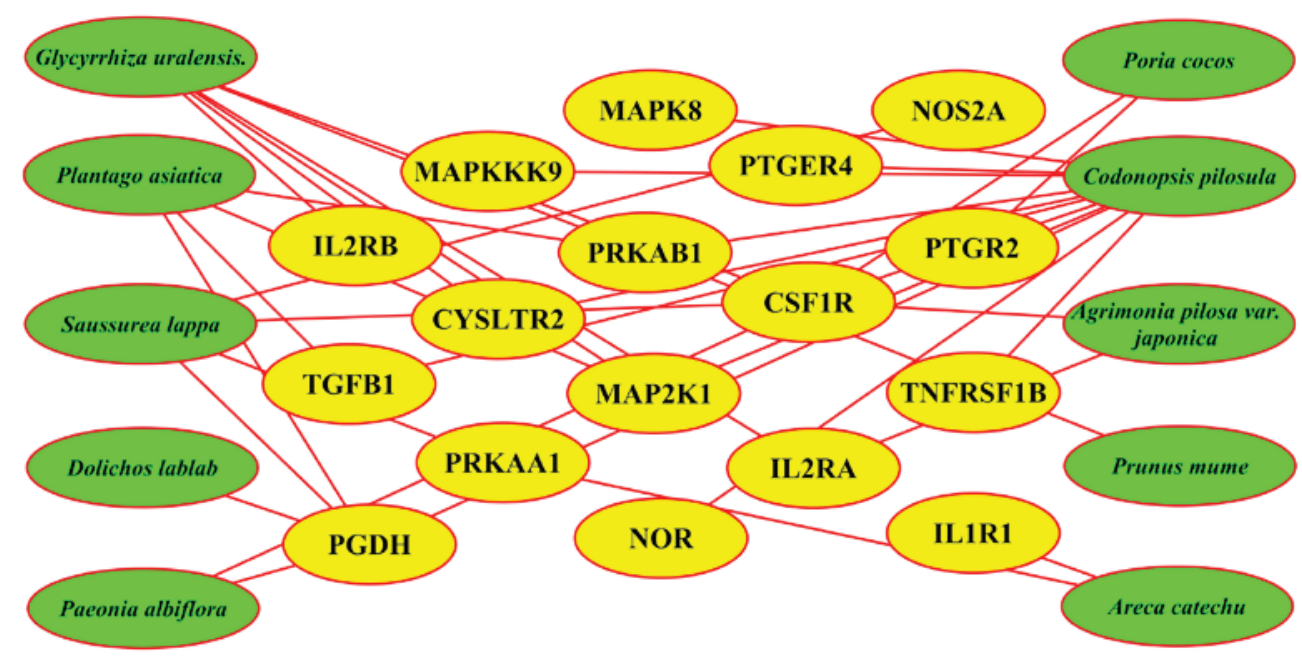

Figure 4. Chinese herbal medicine and target network construction. Green rectangles represent ingredients of JWXJT and the yellow circles represent the screened targets. JWXJT, JiaWeiXianJiTang. 15-PGDH, 15-hydroxyprostaglandin dehydrogenase; PRKAA1, 5'-AMP-activated protein kinase catalytic subunit $\alpha$-1; PRKAB1, 5'-AMP-activated protein kinase subunit $\beta$-1; CYSLTR2, cysteinyl leukotriene receptor 2; MAP2K1, dual specificity mitogen-activated protein kinase kinase 1; IL1R1, interleukin-1 receptor type I; IL2RA, interleukin-2 receptor $\alpha$ chain; IL2RB, interleukin-2 receptor subunit $\beta$; CSF1R, macrophage colony-stimulating factor 1 receptor; MAPK8, mitogen-activated protein kinase 8; MAPKKK9, mitogen-activated protein kinase kinase kinase 9; NOR, nitric oxide reductase; NOS2A, nitric oxide synthase, inducible; PTGER4, prostaglandin E2 receptor EP4 subtype; PTGR2, prostaglandin reductase 2; TGFB1, transforming growth factor $\beta-1$; TNFRSF1B, tumor necrosis factor receptor superfamily member $1 \mathrm{~B}$.

Herb compounds, which exert more effects in 15-PGDH, AMPK, IL-2 and M-CSF-1, and fewer effects in CYSLTR, MAPK, NO and PTGE, may inhibit and reduce the expression levels of inflammatory mediators and inflammatory lesions in tissues. This explains the effect of JWXJT on the immune system, which may be referred to as FuZheng therapy according to TCM. Whereby FuZheng refers to enhancing physical fitness and improving the body's resistance to disease, accompanied by other methods, including appropriate nutrition and functional exercises, so as to overcome disease and restore health.

The key molecule for negative regulation of inflammation is 15 -PGDH. It degrades prostaglandin and antagonizes cyclooxygenase (COX)-2 in vivo (11). AMPK represses expression levels of inflammation inhibitory genes, such as TNF- $\alpha, I L-1 \beta, I L-6$ and inducible nitric oxide synthase (iNOS), reduces the bioactivity of nuclear factor $(\mathrm{NF})-\kappa \mathrm{B}$, promotes the expression of nicotinamide phosphoribosyltransferase and peroxisome-proliferator-activated receptor $\gamma$ coactivator (PGC)-1 $\alpha$, increases $\mathrm{NAD}^{+}$content, and enhances the acetylation enzyme activity of Sirtuin 1 (12-17). In the early stages of IBD, p53 protein overexpression and microsatellite instability are demonstrated, which are early events in the occurrence and progression of $\operatorname{IBD}(18,19)$. However, p53 represses the inflammatory response, which is regulated by NF- $\mathrm{BB}$ (20). The expression of iNOS has been identified to be positively correlated with the expression of hypoxia-inducible factor-1 in IBD patients (21), and a negative correlation was identified between IBD and ILs $(22,23)$. Granulocyte-macrophage colony-stimulating factor (GM-CSF) and TGF are highly expressed in IBD $(24,25)$. GM-CSF therapy reduces inflammation in the colon of mice (26) and TGF- $\beta$ promotes restoration of the intestinal mucosa (27). MAPK, an important regulatory factor, limits inflammatory responses and promotes the resolution of inflammation (28). Furthermore, anti-TNF monoclonal antibody therapy effectively blocks IBD (29). Certain types of TCM treatment enhance the immune system function of IBD patients by reducing TNF- $\alpha$ expression levels (30).

The most common type of chronic intestinal inflammation associated with colorectal cancer is IBD, consisting of $\mathrm{UC}$ and $\mathrm{CD}$. In younger patients, the inflammation is more severe and the development of IBD into colorectal cancer is considered to be more dangerous. Colorectal cancer caused by IBD is referred to as colitis-associated cancer (CAC), which accounts for $15 \%$ of the cause of mortality in IBD patients (31). The study by Kassam et al (31) revealed that the formation of CAC is an 'inflammation-dysplasia-carcinoma' sequential pathological process, and multiple immune cells, cytokines and other immune mediators are involved in the carcinogenesis of colorectal cancer. CAC is predominantly caused by aggravation of IBD by immunological dysfunction, and abnormal activation of the NF- $\mathrm{KB}$ and Janus kinase/signal transducers and activators of transcription signaling pathways. A large quantity of inflammatory cells and pro-inflammation cytokines, such as TNF, IL-6, IL-17 and IL-23, in the intestines activate reactive oxygen species and reactive nitrogen intermediates, and alter biological processes, including cellular growth, apoptosis and proliferation (32). Furthermore, consistent chronic inflammation induces the dysregulation of signaling pathways, such as the Wnt, p53, KRAS and TGF signaling pathways, resulting in tumor development $(19,20)$ Interferon- $\gamma$, TGF- $\beta$ and IL-17 in patients with DC and colon cancer exert inflammatory and immune regulation functions (33). In addition, NF- $\kappa B$, TNF- $\alpha$, IL-1, IL-6, IL8 and IL-27 have significant roles in CAC (34-36). The expression of protease activated receptor-2 (PAR-2) positively correlated with the expression of COX-2 in patients with UC, and the increased expression levels of PAR-2 and COX-2 promoted the occurrence and development of colon cancer by cooperating with 
TF and PGE-2 (37). A previous study demonstrated that granulocyte-colony stimulating factor (G-CSF) expression in a mouse model increased during the course of CAC development. Furthermore, inhibiting the secretion of G-CSF hindered the process of CAC (38).

In conclusion, the network pharmacology analyses revealed that the therapeutic effect of JWXJT on IBD functioned by affecting molecular targets of IBD via the effective compounds of JWXJT, for example, by antagonizing various pathogenic links of IBD, as well as inhibiting inflammatory responses in IBD. In addition, the scientific theory of the TCM, Fuzheng Quxie, used modern biological evidence to explain the TCM principles of monarch, minister, assistant and guide. The present study characterized, in detail, the action mechanism of JWXJT, which included enhancing the immune system and restraining novel blood vessel growth by regulating 15-PGDH, AMPK, IL-2 and M-CSF-1, as well as inhibiting adverse inflammatory reactions. Network pharmacology is able to predict the underlying mechanisms of TCM, and the current results provide a direction for future complex basic biological studies, which will, in part, prevent waste of resources.

\section{Acknowledgements}

The present study was supported by the Natural Science Foundation of China (grant no. 81473628), Fund of Science and Technology Commission of Shanghai (grant no. 14Z1438900), Shanghai Health Development Planning Commission Translational Medicine Focus Issue (grant no. 20124024) and the Natural Science Foundation of China (grant nos. 81573749 and 81303102).

\section{References}

1. Kim TH, Rowat AC and Sloan EK: Neural regulation of cancer: From mechanobiology to inflammation. Clin Transl Immunology 5: e78, 2016.

2. Cooperative Group of Inflammatory Bowel Diseases of Digestive Disease of Chinese Medical Association: Chinese consensus on standard management of inflammatory bowel diseases (2007, Jinan). Chin J Digestion 27: 545-550, 2007.

3. Ye L, Cao Q and Cheng J: Review of inflammatory bowel disease in China. Scientific World Journal. 2013, 2013: 296470. http://dx.doi.org/10.1155/2013/296470.

4. Zhao YJ and Yuan YZ: Clinical study on the relationship between inflammatory bowel disease and colorectal neoplasms J Dig Dis 10: 113-117, 2009.

5. Dhaneshwar SS: Colon-specific prodrugs of 4-aminosalicylic acid for inflammatory bowel disease. World J Gastroenterol 20: 3564-3571, 2014.

6. Zhu LC, Zhu BX, et al: Zhu Lingchun medication experience. Publishing house of Shanghai university of TCM, 1989.

7. Qiu ZJ, Zhu JP and Ma XQ: Zhu Lingchun clinical experience in the treatment of chronic colitis and characteristic, the famous old medical liangchunzhu 19 clinical experience in the series. Liaoning J Tradit Chin Med 28: 399-400, 2001.

8. Zhong LL, Ji W and Wang D: The clinical application of XianJiTang treating of chronic diarrhea, experience prescription of Zhu Liangchun. Jilin J Tradit Chin Med 32: 1098-1099, 2012 (In Chinese).

9. Chen WT, Liu X, Ren JL, et al: Based on cluster analysis of postoperative patients with colorectal cancer medicine research. Chin Arch Tradit Chin Med 1: 101-104, 2016.

10. Shin JH, Jun SL, Hwang SY and Ahn SH: Analytical research to determine the effects of the components of ONGABO on the viability of HepG2 cancer cells by using the sovereign, minister, assistant and courier principle. J Pharmacopuncture 15: 42-51, 2012.
11. Cheng X: Mechanism of COX-2 controling 15-PGDH expression in gastric cancer cells (unpublished PhD thesis). The Fourth Military Medical University, 2010.

12. Giri S, Nath N, Smith B, Viollet B, Singh AK and Singh I: 5-aminoimidazole-4-carboxamide-1-beta-4-ribofuranoside inhibits proinflammatory response in glial cells: A possible role of AMP-activated protein kinase. J Neurosci 24: 479-487, 2004

13. Hattori Y, Suzuki K, Hattori S and Kasai K: Metformin inhibits cytokine-induced nuclear factor kappaB activation via AMP-activated protein kinase activation in vascular endothelial cells. Hypertension 47: 1183-1188, 2006.

14. Zhang K, Deng CS, Zhu YQ and Zhang YM: Significance of nuclear factor-NF- $\kappa \mathrm{B}$, cyclooxygenase 2 and inducible nitric oxide synthase expression in human ulcerative colitis tissues. World Chin J Digestology 10: 575-578, 2002.

15. Minami M, Shimizu K, Okamoto Y, Folco E, Ilasaca ML, Feinberg MW, Aikawa M and Libby P: Prostaglandin E receptor type 4-associated protein interacts directly with NF-kappaB1 and attenuates macrophage activation. J Biol Chem 283: 9692-9703, 2008.

16. Fulco M, Cen Y, Zhao P, Hoffman EP, McBurney MW, Sauve AA and Sartorelli V: Glucose restriction inhibits skeletal myoblast differentiation by activating SIRT1 through AMPK-mediated regulation of Nampt. Dev Cell 14: 661-673, 2008.

17. Jäger S, Handschin C, St-Pierre J and Spiegelman BM: AMP-activated protein kinase (AMPK) action in skeletal muscle via direct phosphorylation of PGC-1alpha. Proc Natl Acad Sci USA 104: 12017-12022, 2007.

18. Li J, Lai MD and Huang Q: Alterations of p53 gene and microsatellite instability in ulcerative colitis and ulcerative colitis-associated colorectal cancer. Zhejiang Da Xue Xue Bao Yi Xue Ban 33: 108-114, 2004 (In Chinese).

19. Wang H, Li Y, Zhang WW and Wang XQ: The expression of $\mathrm{P} 53, \mathrm{~K}-\mathrm{ras}$ and $\mathrm{hMSH} 2$ in ulcerative colitis-associated colorectal cancer. Chin J Digestion 1: 6-9, 2005.

20. Liu G, Park YJ, Tsuruta Y, Lorne E and Abraham E: p53 Attenuates lipopolysaccharide-induced NF-kappaB activation and acute lung injury. J Immunol 182: 5063-5071, 2009.

21. Xu CM, Dong WG, Yu BP, et al: Expression of hypoxia-inducible factor-1alpha (HIF-1 $\alpha$ ) and inducible nitric oxide synthase (iNOS) genes in inflammatory bowel disease. J The Fourth Mil Med Univ 17: 1558-1561, 2004.

22. Xu L: The role of interleukin-2 and platelet activation in the pathogenesis of ulcerative colitis (unpublished $\mathrm{PhD}$ thesis). Fudan University, 2003.

23. Qin Z, Wu C and Chen MM: Ulcerative colitis patients of fecal calcium protein and serum interleukin-2 level and its clinical significance. Guangxi Medical Journal 5: 671-673, 2016.

24 . Wang Y: GM-CSF and IL-1 $\beta$ role in the malignant transformation of chronic enteritis and mechanism research. The Chinese people's liberation army military academy of medical sciences, 2014.

25. Wei WJ, Xu JM, Mei Q and Tang H: Relationship between expression of transforming growth factor- $\beta 1$ and its receptor in colonic mucosa with clinicopathology in patients with ulcerative colitis. Clin J Gastroenterol 13: 341-344, 2008.

26. Lin Y and Cui Y, et al: Application of particle - macrophage colony stimulating factor study curative effect for the treatment of inflammatory bowel disease in mice. Prog Anat Sci 2: 123-126, 2014.

27. Tu P, Zhang XH and Zhou XJ: Updated correlation between angiopoietic factors and inflammatory bowel disease. J Med Postgraduates 20: 1223-1226, 2007.

28. Yin W and Mei ZZ: Dual specificity phosphatase-1: A negative regulator of inflammatory response. Int J Immunol 35: 338-340, 2012.

29. Feng B, Lin ZJ and Bai H: Relationship between anti-tumor necrosis factor- $\alpha$ monoclonal antibody and chronic colitis in mice. Chin J Pract Internal Med 26: 280-282, 2006.

30. Song YY, Ren Y, Liu YZ, Liu W and Liu F: Effect of Chinese medicine on tumor necrosis factor- $\alpha$ in treatment of ul-cerative colitis. China Modern Doctor 14: 81-83, 2015.

31. Kassam Z, Belga S, Roifman I, Hirota S, Jijon H, Kaplan GG, Ghosh S and Beck PL: Inflammatory bowel disease cause-specific mortality: A primer for clinicians. Inflamm Bowel Dis 20: 2483-2492, 2014.

32. Qu N, Xu M, Mizoguchi I, Furusawa J, Kaneko K, Watanabe K, Mizuguchi J, Itoh M, Kawakami Y and Yoshimoto T: Pivotal roles of T-helper 17-related cytokines, IL-17, IL-22, and IL-23, in inflammatory diseases. Clin Dev Immunol, 2013, 2013: 968549. 
33. Li XY: Alteration of the percentage of T helper 17 lymphocytes and interleukin- 6 and -17 expression in elderly colorectal cancer patients (unpublished PhD thesis). Dalian Medical University, 2014.

34. Yang H: NF- $\mathrm{B} / \mathrm{IL}-6$ pathway in relation to the colitis associated colorectal cancer development (unpublished $\mathrm{PhD}$ thesis). Zhengzhou University, 2014.

35. Feng DM: Determination and clinical significance of sIL-2R, IL-8 and TNF-alpha in the inflammatory bowel disease colorectal cancer (unpublished $\mathrm{PhD}$ thesis). Yanbian University, 2013.

36. Cui Y, Liu ZJ, Zhao ZG, Zheng P-Y and Huang D-F: Significance of interleukin-27 expression in the intestinal mucosa of patients with inflammatory bowel disease. World Chin J Digestology 1: 39-43, 2010.
37. Ma X, Xie Yk, Liu T, Zhang Q, Wang CH, Zhou CJ and Gao YJ: Relative research between PAR-2 and COX-2 expressed in intestinal mucosa of patients with ulcerative colitis. Chin J Curr Adv Gen Surg 2: 107-111, 2013.

38. Shi XY, Yuan W, Tang WY, et al: The expression of G-CSF in colitis-associated cancer. Oncology Progress 2: 168-171, 174, 2016. 